\title{
Verhaltensanalyse in der Verhaltenstherapie
}

\author{
Abbruzzese, Elvira ; Kübler, Ulrike
}

\begin{abstract}
Conducting a behavior analysis is a common diagnostic procedure in behavior therapy. It provides a dysfunction-independent working model for the behavioral and multimodal exploration of the patient's condition as well as subsequently an important basis for the preparation of a case concept. Starting from a definition of behavior analysis the components of a behavior analysis as well as their differentiations are introduced. Subsequently, a well-established practical procedure of a behavior analysis is exemplarily demonstrated by means of a case study. Finally, the current significance of this technique is discussed for therapeutic purposes.
\end{abstract}

DOI: https://doi.org/10.1159/000352030

Posted at the Zurich Open Repository and Archive, University of Zurich ZORA URL: https://doi.org/10.5167/uzh-85956

Journal Article

Published Version

Originally published at:

Abbruzzese, Elvira; Kübler, Ulrike (2013). Verhaltensanalyse in der Verhaltenstherapie. Verhaltenstherapie, 23(2):108-116.

DOI: https://doi.org/10.1159/000352030 


\title{
Verhaltensanalyse in der Verhaltenstherapie
}

\author{
Elvira Abbruzzese Ulrike Kübler \\ Klinische Psychologie und Psychotherapie, Universität Zürich, Schweiz
}

\section{Schlüsselwörter}

Verhaltensanalyse - Mikroanalyse - S-O-R-K-C-Modell .

Verhaltenstherapie · Diagnostik

\section{Zusammenfassung}

Die Verhaltensanalyse ist ein weitverbreitetes diagnostisches Verfahren in der Verhaltenstherapie. Sie gibt ein störungsübergreifendes Raster zur verhaltensnahen und multimodalen Exploration der Problematik des Patienten vor und liefert in Folge dessen eine wichtige Grundlage für die Ausarbeitung der Fallkonzeption. Nach einer Begriffsdefinition von Verhaltensanalyse, in deren Rahmen die Bestandteilteile der Verhaltensanalyse mit ihren Erweiterungen vorgestellt werden, wird in dem aktuellen Beitrag praxisnah das derzeit etablierte und interventionsbezogene Vorgehen bei einer Verhaltensanalyse beschrieben. Abschließend wird die gegenwärtige Bedeutung des Verfahrens für den therapeutischen Prozess diskutiert.

\section{Einführung}

In der Verhaltenstherapie sind Diagnostik und Intervention eng miteinander verbunden. Ein weitverbreitetes diagnostisches Verfahren in der Verhaltenstherapie ist die Verhaltensanalyse. Die Verhaltensanalyse gibt ein störungsübergreifendes Raster zur verhaltensnahen und multimodalen Exploration der Problematik des Patienten vor und liefert in Folge dessen eine wichtige Grundlage für die Ausarbeitung der Fallkonzeption. Seit den 1990er Jahren jedoch steht die Verhaltensanalyse trotz ihres hohen Bekanntheitsgrads zusehends in der Kritik - dies nicht zuletzt aufgrund der sich fortwährend entwickelnden klassifikatorischen Dia-

\author{
Keywords \\ Behavior analysis - Microanalysis - S-O-R-K-C model . \\ Behavior therapy · Diagnostics
}

\section{Summary}

\section{Behavior Analysis in Behavior Therapy}

Conducting a behavior analysis is a common diagnostic procedure in behavior therapy. It provides a dysfunction-independent working model for the behavioral and multimodal exploration of the patient's condition as well as subsequently an important basis for the preparation of a case concept. Starting from a definition of behavior analysis the components of a behavior analysis as well as their differentiations are introduced. Subsequently, a well-established practical procedure of a behavior analysis is exemplarily demonstrated by means of a case study. Finally, the current significance of this technique is discussed for therapeutic purposes.

\begin{tabular}{ll}
\hline KARGER & ( ) 2013 S. Karger GmbH, Freiburg \\
1016-6262/13/0232-0108\$38.00/0 \\
$\begin{array}{l}\text { Fax +497614520714 } \\
\text { Information@Karger.com } \\
\text { www.karger.com }\end{array}$ & $\begin{array}{l}\text { Accessible online at: } \\
\text { www.karger.com/ver }\end{array}$
\end{tabular}




\section{Definition und Funktion der Verhaltensanalyse}

Der Terminus «Verhaltensanalyse» wird uneinheitlich verwendet. Nach Schulte [Schulte, 1996; Schulte, 1999] wird die Verhaltensanalyse als Teil der Problemanalyse definiert, die neben der Verhaltensanalyse zusätzlich die Problemstrukturierung umfasst. Während im Rahmen der Problemstrukturierung Teilprobleme (z.B. Panikattacken) durch die Formulierung von Ist- und Soll-Zuständen beschrieben werden, wird mit der sich daran anschließenden Verhaltensanalyse das Ziel verfolgt, jene Bedingungen $\mathrm{zu}$ identifizieren, die die vorläufig definierten Teilprobleme bzw. das problematische Verhalten mitbestimmen und aufrechterhalten. Die Funktion der Verhaltensanalyse ist demnach die Ausarbeitung eines hypothetischen Bedingungsmodells, das das Problemverhalten in Verbindung mit den auslösenden sowie aufrechterhaltenden Bedingungen beschreibt, um so eine Basis für die Auswahl geeigneter Interventionsmethoden zu schaffen. In diesem Sinne bezeichnet der Begriff Verhaltensanalyse die Diagnostik der funktionalen Zusammenhänge des Problemverhaltens mit den vorausgehenden und nachfolgenden Bedingungen [vgl. Knafla und Ehlert, 2001, S. 146]. Der Verhaltensbegriff umfasst hierbei sowohl offen beobachtbare Verhaltensäußerungen als auch Kognitionen sowie physiologische und emotionale Reaktionen.

Historisch betrachtet handelt es sich bei der Verhaltensanalyse um einen auf lerntheoretischen Grundannahmen basierenden diagnostischen Ansatz. Entsprechend dieser Tradition wurde das Problemverhalten (R) zunächst ausschließlich als Funktion der unmittelbar vorausgehenden situativen Stimuli (S) und nachfolgenden Konsequenzen (C) betrachtet (vgl. Skinner'sche S-R-C-Verhaltensformel). Mit der Einführung der Kontingenzvariable (K) durch Lindsley [1964] sowie der Organismusvariable (O) durch Kanfer und Saslow [1969] wurde das S-R-C-Modell allerdings schon bald zu dem bis heute im Wesentlichen gebräuchlichen S-O-R-K-C-Modell erweitert. Da eine Verhaltensanalyse basierend auf dem S-O-RK-C-Modell das Problemverhalten in einer konkreten Situation auf einem zeitlichen Kontinuum betrachtet, wird diese Form der Verhaltensanalyse auch als Mikroanalyse oder horizontale Verhaltensanalyse bezeichnet. Der bis heute fortwährende Gebrauch des S-O-R-K-C-Modells als diagnostisches Instrument in der verhaltenstherapeutischen Praxis soll allerdings nicht darüber hinwegtäuschen, dass die einzelnen Modellvariablen im Laufe ihrer Entwicklungsgeschichte zunehmend ausdifferenziert wurden. Eine bedeutsame Erweiterung und Ausdifferenzierung der O-Variable, die ursprünglich nur biologische Ursachen des Problemverhaltens umfasste, nahmen beispielsweise Bartling und Kollegen [1980] in der Zeit der kognitiven Wende vor: Sie reicherten die O-Variable in ihrem Modell der Verhaltensanalyse (vgl. Verhalten in der Situation, ViS) um Faktoren der individuellen Wahrnehmung und der inneren Reizverarbeitung an. Die Autoren nehmen an, dass dem Problemverhalten ein innerer, weitgehend implizit ablaufender Informationsverarbeitungsprozess vorausgeht.
Dieser Informationsverarbeitungsprozess umfasst zum einen den Wahrnehmungsprozess (WP), der als Selektionsprozess mit den Anteilen Orientierung, Aufnehmen und Kodieren von Informationen zu thematisieren ist, und zum anderen die innere Verarbeitung (iV), die ihrerseits aus einer weiteren Phase der Informationsverarbeitung und der Handlungsvorbereitung besteht (weiterführend siehe z.B. Bartling et al. [2008]).

Mit Einführung der sogenannten vertikalen Verhaltensanalyse durch Grawe [Grawe, 1982] wurde die verhaltensanalytische Diagnostik darüber hinaus um eine neue Sichtweise erweitert. Nach dieser Sichtweise ist das Problemverhalten als Funktion von individuellen überdauernden und verhaltenssteuernden Zielen und Plänen zu betrachten [vgl. Zaby und Heider, 2012, S. 294]. Die vertikale Verhaltensanalyse wird auch als Makroanalyse bezeichnet. Zu den Weiterentwicklungen der vertikalen Verhaltensanalyse zählt die Plananalyse [Grawe und Caspar, 1984], auf deren Ausarbeitung in diesem Beitrag nicht näher eingegangen wird. Interessierte Leser seien auf entsprechende Literatur verwiesen (z.B. Caspar und Goldfried [2007]). Stattdessen beziehen wir uns nachfolgend auf das verhaltensanalytische Vorgehen nach dem S-O-R-KC-Modell - ein Modell, das sich nach Ansicht verschiedener klinisch tätiger Autoren nach wie vor sehr gut zur Strukturierung von diagnostischen Informationen eignet [Caspar, 1996; Hautzinger, 2011; Tuschen-Caffier und von Gemmeren, 2009]. Wir berücksichtigen dabei das gegenwärtig übliche Ausdifferenzierungsniveau der einzelnen Modellvariablen.

\section{Das S-O-R-K-C-Modell}

Im Kontext der Verhaltenstherapie wird angenommen, dass jedes (Problem-)Verhalten durch ein zunächst unbekanntes Netz von Bedingungen ausgelöst und aufrechterhalten wird [Kanfer et al., 2006]. Diese Bedingungen gilt es während des diagnostischen Prozesses sukzessive aufzudecken, sodass auf Basis des erarbeiteten Bedingungsmodells schließlich eine adäquate Behandlungsstrategie festgelegt werden kann. Die Verhaltensanalyse nach dem S-O-R-K-C-Modell analysiert das Problemverhalten in einer konkreten Situation (d.h. einschließlich der Bedingungen, die dem Verhalten vorausgehen und folgen) und stellt somit ein probates Mittel zur Ausarbeitung eines Bedingungsmodells dar (Abb. 1). Um die problemrelevanten Bedingungen möglichst eindeutig zu bestimmen, empfiehlt es sich, das Problemverhalten in unterschiedlichen Situationen zu analysieren, d.h. mehrere Verhaltensanalysen durchzuführen.

\section{Leitfaden für die Durchführung}

Für die Durchführung einer Verhaltensanalyse nach dem S-O-R-K-C-Modell empfehlen Tuschen-Caffier und von Gemmeren [2009] folgendes Vorgehen: 
Schritt 1: Beschreibung des im Vorfeld mit dem Patienten definierten Problemverhaltens (Reaktion, R).

Schritt 2: Identifikation der vorausgehenden, problemrelevanten Ereignisse (Stimulus, S).

\section{SORKC Modell}

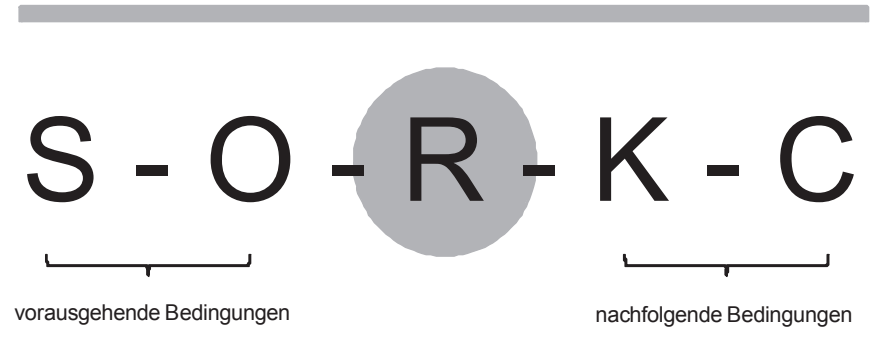

Abb. 1. Horizontale Verhaltensanalyse nach dem S-O-R-K-C-Modell (nach Neudeck und Mühlig [2013]).
Schritt 3: Analyse der auf das Problemverhalten folgenden Konsequenzen («consequences», C).

Schritt 4: Exploration situationsübergeifernder, problemrelevanter Faktoren des Patienten (Organismus, O).

Nachfolgend werden die einzelnen Schritte bzw. die Variablen des S-O-R-K-C-Modells ausführlicher erläutert. Begleitend hierzu sind in Tabelle 1 die Modellvariablen und ihre gängigen Abkürzungen aufgelistet.

\section{Schritt 1 «Verhaltensvariable $R »$}

$\mathrm{Zu}$ Beginn einer Verhaltensanalyse besteht das Ziel darin, das unerwünschte Problemverhalten (R-Variable; z.B. Essanfälle oder Panikattacken) in einer konkreten Situation auf verschiedenen Verhaltensebenen möglichst präzise zu beschreiben. $\mathrm{Zu}$ diesen Ebenen zählen die motorische («Was haben Sie konkret gemacht?»), die kognitive («Was haben Sie dabei gedacht?»), die emotionale («Wie haben Sie sich wäh-

Tab. 1. Das S-O-R-K-C-Modell der Verhaltensanalyse (nach Meinlschmidt und Tegethoff [2009])

\begin{tabular}{|c|c|c|}
\hline Gängige Abkürzungen & Komponente & Erklärung \\
\hline \multirow{4}{*}{$\begin{array}{l}\mathrm{S}_{\mathrm{i}} \\
\mathrm{S}_{\mathrm{e}} \\
\mathrm{S}^{\mathrm{D}}\end{array}$} & Stimulus & $\begin{array}{l}\text { Stimuli, die in der problemrelevanten Situation auf den } \\
\text { Organismus einwirken }\end{array}$ \\
\hline & interner Stimulus & \\
\hline & externer Stimulus & \\
\hline & $\begin{array}{l}\text { diskriminativer Stimulus, der mit Verstärkung } \\
\text { assoziiert ist }\end{array}$ & \\
\hline $\mathrm{S}^{\Delta}$ & $\begin{array}{l}\text { diskriminativer Stimulus, der mit Extinktion, } \\
\text { d.h. dem Ausbleiben von Verstärkung assoziiert ist }\end{array}$ & \\
\hline CS & konditionierter Stimulus & \\
\hline UCS & unkonditionierter Stimulus & \\
\hline $\mathrm{O}$ & Organismus & $\begin{array}{l}\text { Situationsübergreifende biologische und psychologische } \\
\text { Personenmerkmale, die das Problemverhalten } \\
\text { begünstigen; die O-Variable bildet die Schnittstelle zur } \\
\text { vertikalen Verhaltensanalyse }\end{array}$ \\
\hline $\mathrm{R}$ & Reaktion & $\begin{array}{l}\text { Problemverhalten auf kognitiver, emotionaler, } \\
\text { physiologischer und motorischer Ebene }\end{array}$ \\
\hline $\mathrm{R}_{\text {kogn }}$ & kognitive Reaktion & \\
\hline $\mathrm{R}_{\mathrm{emot}}$ & emotionale Reaktion & \\
\hline $\mathrm{R}_{\text {phys }}$ & physiologische Reaktion & \\
\hline $\mathrm{R}_{\mathrm{mot}}$ & offen beobachtbare Reaktion & \\
\hline $\mathrm{K}$ & Kontingenz & $\begin{array}{l}\text { Regelhaftigkeit, mit der eine bestimmte Konsequenz auf } \\
\text { ein bestimmtes Verhalten folgt (z.B. immer oder } \\
\text { intermittierend) }\end{array}$ \\
\hline $\mathrm{C}$ & Konsequenz & Veränderung, die auf das Problemverhalten folgt \\
\hline \multicolumn{3}{|l|}{ Konsequenzarten } \\
\hline $\mathrm{C}^{+}$ & Eintreten einer positiven Konsequenz & positive Verstärkung \\
\hline $\mathrm{C}^{-}$ & Eintreten einer negativen Konsequenz & direkte Bestrafung Typ 1 \\
\hline $\mathrm{C}^{+}$ & Wegfall einer positiven Konsequenz & indirekte Bestrafung Typ 2 / Löschung \\
\hline $\mathrm{C}^{-}$ & Wegfall einer negativen Konsequenz & negative Verstärkung \\
\hline \multicolumn{3}{|l|}{ Zeitpunkt } \\
\hline $\mathrm{C}_{\mathrm{k}}$ & kurzfristige Konsequenz & \\
\hline $\mathrm{C}_{1}$ & langfristige Konsequenz & \\
\hline \multicolumn{3}{|l|}{ Entstehungsort } \\
\hline $\mathrm{C}_{\mathrm{i}}$ & interne Konsequenz & \\
\hline $\mathrm{C}_{\mathrm{e}}$ & externe Konsequenz & \\
\hline
\end{tabular}


renddessen gefühlt?») sowie die physiologische Ebene («Welche körperlichen Veränderungen haben Sie währenddessen wahrgenommen?»).

\section{Schritt 2 «Stimulusvariable S»}

In einem weiteren Schritt wird die S-Variable erhoben. Unter $\mathrm{S}$ werden alle externen $\left(\mathrm{S}_{\mathrm{i}}\right)$ und internen Bedingungen $\left(\mathrm{S}_{\mathrm{e}}\right)$ zusammengefasst, die dem Problemverhalten vorausgehen und mit diesem potenziell in funktionalem $\mathrm{Zu}$ sammenhang stehen. $\mathrm{Zu}$ den externen Bedingungen zählen physikalische Umweltfaktoren (z.B. erhöhte Raumtemperatur) und soziale Merkmale (z.B. An-/Abwesenheit / kritische Bemerkungen anderer Personen). Die internen Bedingungen umfassen mitunter Gedanken (z.B. «Ich schaffe das nicht»), Gefühle (z.B. Niedergeschlagenheit) und körperliche Veränderungen (z.B. Anspannung). Die Beschreibung der Situationsmerkmale bzw. der S-Variable sollte möglichst detailliert erfolgen. Neben der Differenzierung $S_{i} / S_{e}$ empfehlen sich Angaben zur Stimulusqualität, die aus der Art des funktionalen Zusammenhangs zwischen S und R abgeleitet werden können. Der Zusammenhang zwischen S und R kann klassisch konditioniert, unkonditioniert (d.h. reflektorisch, biologisch determiniert) oder durch Reizdiskriminationslernen vermittelt sein. Entsprechend der Art des Zusammenhangs erhält S die Qualität eines konditionierten Stimulus (CS), eines unkonditionierten Stimulus (UCS) oder eines diskriminativen Hinweisreizes. Als diskriminativer Hinweisreiz kann S das Problemverhalten sowohl fördern oder hervorrufen $\left(S^{D}\right)$ als auch hemmen und verhindern $\left(\mathrm{S}^{\Delta}\right)$ [Hautzinger, 2011].

\section{Schritt 3 «Konsequenzvariable $C »$ und «Kontingenz- variable $K »$}

Die Verhaltenskonsequenzen können den Zeitpunkt, die Frequenz, die Intensität, die Dauer sowie die Stabilität des Problemverhaltens beeinflussen [Neudeck und Mühlig, 2013]. Demgemäß besteht bei der Analyse der C-Variable das Ziel darin, diejenigen auf das Problemverhalten folgenden Ereignisse $\mathrm{zu}$ identifizieren, die im Sinne operanter Lernprozesse eine verhaltenssteuernde Funktion ausüben. Die Beschreibung der Konsequenzen erfolgt üblicherweise auf 3 Dimensionen:

1. Zeitpunkt des Eintretens: kurzfristige Konsequenz $\left(\mathrm{C}_{\mathrm{k}}\right.$; hohe Kontiguität, d.h. die Konsequenz erfolgt unmittelbar auf das Verhalten) versus langfristige Konsequenz $\left(\mathrm{C}_{\mathrm{l}}\right.$; geringe Kontiguität, d.h. die Konsequenz tritt zeitlich verzögert auf)

2. Entstehungsort: interne Konsequenz $\left(C_{i}\right.$; intern entstehend, z.B. Selbstbewertung oder körperliche Reaktion) versus externe Konsequenz $\left(\mathrm{C}_{\mathrm{e}}\right.$; aus der Umwelt stammend, z.B. Aufmerksamkeit erfahren)

3. Qualität der Konsequenz: positive Verstärkung $\left(\mathrm{C}^{+}\right.$; Eintreten einer positiven Konsequenz; z.B. Aufmerksamkeit erfahren) versus negative Verstärkung ( $\mathrm{C}^{=} ;$Wegfall einer negativen Konsequenz, z.B. Anspannung lässt nach); direkte Bestrafung $\left(\mathrm{C}^{-}\right.$; Eintreten einer negativen Konsequenz, z.B. Strafarbeit) versus indirekte Bestrafung $\left(\Theta^{+}\right.$; Wegfall einer positiven Konsequenz, z.B. Reduktion der Aufmerksamkeit).

Die Forschungsergebnisse zum operanten Konditionieren zeigen, dass die kurzfristigen Konsequenzen im Vergleich zu den langfristigen Konsequenzen deutlich verhaltenswirksamer sind [Evans et al., 1978; Lundberg et al., 1975]. Entsprechend bedeutsam ist die Suche nach den kurzfristen Konsequenzen des Problemverhaltens.

Während der Analyse der C-Variable bietet sich zugleich die Analyse der K-Variable an. Die K-Variable gibt Auskunft darüber, mit welcher Regelmäßigkeit eine bestimmte Konsequenz auf ein bestimmtes Problemverhalten folgt. Dabei gilt: Je öfter eine bestimmte Konsequenz auf ein bestimmtes Problemverhalten folgt, desto höher die Kontingenz. Informationen zur Kontingenz erlauben eine Aussage über die Stabilität bzw. den Löschungswiderstand eines Problemverhaltens. Liegt eine geringe Kontingenz zwischen dem Problemverhalten und dem Verstärker vor, ist die Stabilität bzw. der Löschungswiderstand des Problemverhaltens hoch. Gleiches gilt darüber hinaus für eine geringe Kontiguität, d.h. tritt der Verstärker zeitlich verzögert auf, ist die Stabilität des Problemverhaltens als hoch einzustufen. Liegt demgegenüber sowohl eine hohe Kontiguität als auch eine hohe Kontingenz zwischen dem Problemverhalten und dem Verstärker vor, ist die Stabilität bzw. der Löschungswiderstand des Problemverhaltens gering [Narciss, 2011].

\section{Schritt 4 «Organismusvariable O»}

Abschließend ist die O-Variable zu explorieren, die als eine vermittelnde Bedingung zwischen der vorausgehenden Situation und dem Problemverhalten zu betrachten ist. Darüber hinaus kann die O-Variable als Schnittstelle zwischen der horizontalen und vertikalen Verhaltensanalyse angesehen werden [Zaby und Heider, 2012]. In die O-Variable werden alle biologisch-physiologischen und psychosozialen Faktoren des Patienten aufgenommen, die im Sinne von zeit- und situationsstabilen Persönlichkeitseigenschaften problemrelevant sind. Hierzu zählen körperliche Erkrankungen, genetische Prädispositionen, physiologische Zustände, Einstellungen, Kontroll- und Grundüberzeugungen, Schemata und Persönlichkeitsmerkmale, Intelligenz und Selbstkonzept.

Eine praxisnahe Veranschaulichung des Vorgehens bei der Verhaltensanalyse nach dem S-O-R-K-C-Modell erfolgt im Anschluss an diesen Abschnitt anhand eines Fallbeispiels.

\section{Hilfsmittel zur Informationserhebung}

Die Hilfsmittel zur Gewinnung der Informationen für die Verhaltensanalyse nach dem S-O-R-K-C-Modell sind vielfältig und umfassen mitunter Selbst- und Fremdberichte über das Verhalten und die Konsequenzen in problemrelevanten Situationen, Beobachtungsverfahren für das konkrete Verhalten in der Situation, Verhaltenstests, Rollenspiele sowie Fra- 
Tab. 2. Lerntheoretisch begründete Möglichkeiten der Verhaltensmodifikation über die Variablen des S-O-R-K-C-Modells (nach Neudeck und Mühlig [2013])

\begin{tabular}{|c|c|c|c|}
\hline & Verhaltensaufbau & Verhaltensabbau & Verhaltensstabilisierung \\
\hline S & $\begin{array}{l}\text { klassische Konditionierung; } \\
\text { Sensibilisierung }\end{array}$ & $\begin{array}{l}\text { Gegenkonditionierung, d.h. verhaltens- } \\
\text { auslösenden Reiz mit alternativem } \\
\text { Verhalten verknüpfen; Desensibilisierung, } \\
\text { d.h. Signalwirkung des Reizes durch } \\
\text { schrittweise Konfrontation abbauen }\end{array}$ & $\begin{array}{l}\text { «Fading out», d.h. zunehmendes } \\
\text { Ausblenden situativer Bedingungen; } \\
\text { Generalisierung von Reizen }\end{array}$ \\
\hline $\mathrm{R}$ & $\begin{array}{l}\text { «Shaping», d.h. Aufbau eines neuen } \\
\text { Verhaltens durch sukzessiven Aufbau von } \\
\text { Verhaltenselementen; } \\
\text { «Chaining», d.h. Verkettung bekannter } \\
\text { Verhaltensweisen zu einer komplexen } \\
\text { Reaktion }\end{array}$ & $\begin{array}{l}\text { Sättigung, d.h. Präsentation des } \\
\text { unerwünschten Verhaltens als oft zu } \\
\text { wiederholende Aufgabe }\end{array}$ & $\begin{array}{l}\text { Übung und systematisches Variieren } \\
\text { der Übungsformen, Übungsmethoden } \\
\text { und Übungsbedingungen }\end{array}$ \\
\hline K & $\begin{array}{l}\text { hohe Kontingenz und Kontiguität sowohl } \\
\text { für Konsequenzen als auch Hinweisreize }\end{array}$ & $\begin{array}{l}\text { Auflösung von hoher Kontingenz und/oder } \\
\text { Kontiguität }\end{array}$ & $\begin{array}{l}\text { Reduktion von hoher Kontingenz } \\
\text { und/oder Kontiguität }\end{array}$ \\
\hline $\mathrm{C}$ & positive und negative Verstärkung & direkte und indirekte Bestrafung & \\
\hline
\end{tabular}

gebögen, Listen und Inventare zur Erfassung von zeit- und situationsstabilen Persönlichkeitseigenschaften, situativen Parametern und Verstärkern [Hautzinger, 2011].

\section{Möglichkeiten der Verhaltensmodifikation}

Um die Nützlichkeit einer guten Verhaltensanalyse bzw. eines guten Bedingungsmodells für die Auswahl adäquater therapeutischer Methoden zu verdeutlichen, sind in Tabelle 2 lerntheoretisch begründete Möglichkeiten der Verhaltensmodifikation über die Variablen des S-O-R-K-C-Modells zusammengestellt.

\section{Fallbeispiel}

Anhand des nachfolgenden Fallbeispiels soll der Einsatz der Verhaltensanalyse im therapeutischen Prozess erläutert werden. Die Analyse des spezifischen Problemverhaltens, die zur diagnostischen Beurteilung herangezogen werden kann, sowie die daraus resultierenden Therapieziele und konkreten Behandlungsschritte, die sich aus der Verhaltensanalyse ableiten lassen, sollen daran aufgezeigt werden.

\section{Behandlungsanlass und spontan berichtete Symptomatik des Patienten}

Der Patient ist zum Zeitpunkt des Erstkontakts im psychiatrischen Ambulatorium 26 Jahre alt, verheiratet und Vater eines 2-jährigen Kindes. Die Lebenspartnerin des Patienten hat ihn zur psychotherapeutischen Behandlung überredet, da der Leidensdruck zusehends zunehme. Der Patient berichtet, dass ihn seine Arbeit als Biologe bei einer Pharmafirma überfordere. Grundsätzlich sei es ihm sehr wichtig, dass er seine Arbeit perfekt und fehlerfrei verrichte. Er leide bereits seit Jahren unter einem Waschzwang, der die letzten Monate zugenommen habe. Durch das viele Waschen seien seine Hände häufig stark gerötet, jucken, zeigen Ekzeme und bluten bis- weilen sogar. In den letzten Jahren sei seine Stimmung immer schlechter geworden. Er fühle sich häufig bedrückt, ausgelaugt und müde. Seit einigen Wochen habe der Patient das Gefühl, er dekompensiere langsam. Der Waschzwang sei aus einer großen Angst vor einer Kontamination mit Würmern entstanden: Er habe als Jugendlicher nach einem kurzen Auslandsaufenthalt eine Wurminfektion erlitten. Damals habe er sich ausführlich über Wurmerkrankungen informiert und gelesen, dass es Parasiten gäbe, bei denen man erst Jahre nach der Infektion merke, dass man krank sei und dass man letztlich unweigerlich daran sterbe. Diese Vorstellung, regelrecht von innen aufgefressen zu werden, löse in ihm einen enormen Ekel aus. Er müsse sich daher immer vergewissern, dass alles sauber sei. Er könne zur Zeit weder Salat noch rohes Gemüse essen, weil er nie sicher sei, ob die Nahrungsmittel sauber gewaschen seien. Er habe bereits früh von seiner Mutter gelernt wie wichtig es sei, hygienisch zu sein. Bereits als Kind habe er sich die Hände vor dem Essen ausgiebig gewaschen und desinfiziert. Bei ihnen zu Hause sei es immer äußerst sauber gewesen. Umso mehr habe ihn damals die Wurmerkrankung im Jugendalter beelendet, da er bis heute denke, dass er diese durch ein hygienischeres Verhalten hätte vermeiden können.

\section{Diagnostische Beurteilung nach DSM-IV (ICD-10)}

Aufgrund der vom Patienten geschilderten Symptomatik sind gemäß des DSM-IV die Kriterien für eine Zwangsstörung wie auch für eine spezifische Phobie (in diesem Falle eine Mysophobie) gegeben. Zusätzlich kann eine reaktive, mittelgradig depressive Episode kodiert werden (Tab. 3).

\section{Die Erarbeitung der Verhaltensanalyse}

Nach einer ersten Anamnese können bereits gemeinsam mit dem Patienten erste Verhaltensanalysen durchgeführt werden. Dabei werden die Verkettung von dysfunktionalen Annahmen, Schemata sowie der spezifischen Phobien betreffenden Problemverhaltensweisen, Gedankeninhalten und 


\begin{tabular}{ll}
\hline Achse I & $\begin{array}{l}\text { 300.3 Zwangsstörung } \\
\text { (ICD-10 F42.2 Zwangsstörung mit Zwangsgedanken und -handlungen, gemischt) } \\
\text { 300.29 Spezifische Phobie (Mysophobie) }\end{array}$ \\
& (ICD-10 F40.2 spezifische Phobie) \\
& 296.22 Major Depression, einzelne Episode, mittelschwer \\
& (ICD-10 F32.1 mittelgradige depressive Episode) \\
& - \\
Achse II & - \\
Achse III & anamnestisch mütterliche Überfürsorglichkeit (ICD-10 Z62.1) \\
Achse IV & globale Erfassung des Funktionsniveaus GAF: 63 \\
Achse V &
\end{tabular}

Tab. 4. Beispiel einer Verhaltensanalyse (vgl. dazu Tab. 1)

\begin{tabular}{|c|c|c|}
\hline Stimulus & $\mathrm{S}_{\mathrm{e}}$ & $\begin{array}{l}\text { Der Patient ist mit seiner Ehefrau in einem Lebensmittelladen und steht vor der Obstauslage. Gerne würde er } \\
\text { sich gesünder ernähren. Er berührt flüchtig eine Schachtel mit Erdbeeren }\end{array}$ \\
\hline O-Variable & & $\begin{array}{l}\text { Grundannahmen und Erwartungen: } \\
\text { «Die Wahrscheinlichkeit, mich mit einem Fuchsbandwurm zu infizieren, wenn ich die Erdbeeren berühre, } \\
\text { ist groß» } \\
\text { «Nur wenn ich die Erdbeeren ganz extrem gründlich wasche, kann ich die Gefahr einer Infektion } \\
\text { einigermaßen kontrollieren» } \\
\text { Überdauernde Persönlichkeitsmerkmale: } \\
\text { Veranlagung, perfektionistisch zu sein } \\
\text { Vorübergehende und überdauernde physiologische Zustände: } \\
\text { Starkes physiologisches Arousal bei Gedanken an Würmer }\end{array}$ \\
\hline Reaktion & $\begin{array}{l}\mathrm{R}_{\text {kogn }} \\
\mathrm{R}_{\text {emot }} \\
\mathrm{R}_{\text {phys }} \\
\mathrm{R}_{\text {mot }}\end{array}$ & $\begin{array}{l}\text { «Diese Erdbeeren sind schmutzig! Sie sind nahe an der Erde gewachsen. Da könnte ich mich mit Würmern } \\
\text { infizieren, die mich von innen her zerfressen!» } \\
\text { Ekel, starke Angst und Aggression } \\
\text { Anspannung, erhöhter Puls, Schwitzen, erhöhte Muskelanspannung im Nacken- und Rückenbereich } \\
\text { Er säuberte sich die Hände mit einem Papiertaschentuch und versucht, sich nicht mehr zu berühren } \\
\text { (Schadensbegrenzung, aber nur unzureichende Beruhigung bis er die Hände ausführlich waschen und } \\
\text { desinfizieren kann) }\end{array}$ \\
\hline Konsequenzen & $\begin{array}{l}\mathrm{C}_{\mathrm{ki}}: \\
\mathrm{C}= \\
\mathrm{C}_{\mathrm{ke}}: \\
\mathrm{C}- \\
\mathrm{C}_{\mathrm{li}}: \\
\mathrm{C}-\end{array}$ & $\begin{array}{l}\text { Die Beziehung zu seiner Ehefrau wird aufgrund von situationsbezogenen Auseinandersetzungen beeinträchtigt } \\
\text { Handekzeme aufgrund des häufigen Händewaschens } \\
\text { Beeinträchtigung im Alltag; gesunde Ernährung mit rohen Früchten und Gemüse wird erschwert }\end{array}$ \\
\hline
\end{tabular}

Gefühlen ersichtlich. Es ist zu beachten, dass der Patient in diesem Fallbeispiel 3 komorbide Störungen aufweist, welche jedoch stark miteinander in Beziehung stehen, da der Waschzwang, wie bereits weiter oben erwähnt, als aktive Vermeidungsstrategie im Rahmen seiner Erkrankungsphobie (Mysophobie) gesehen werden kann, während die depressive Symptomatik mit großer Wahrscheinlichkeit als Reaktion auf die starke Einschränkung im Alltag durch die ersten beiden Störungskomplexe entstanden ist.

Die Erarbeitung von Verhaltensanalysen zu diesem Zeitpunkt der Therapie bietet dem Patienten die Möglichkeit, ein individuelles Funktionsmodell zu etablieren, welches ihm Zusammenhänge seiner eigenen innerpsychischen Dynamik und des daraus resultierenden Verhaltens zugänglich macht. Gleichzeitig dient die Verhaltensanalyse als hypothetisches Bedingungs- bzw. Arbeitsmodell, das es zu überprüfen gilt. Wichtig dabei erscheint die Tatsache, dass das Modell für den Patienten nachvollziehbar und authentisch ist. Kann sich der Patient nicht mit der Verhaltensanalyse identifizieren, ist diese überflüssig. Das Erarbeiten von Verhaltensanalysen im Therapiesetting kommt einem Üben gleich, das es dem Patienten langfristig erleichtern kann, sein eigenes Verhalten auch im Alltag besser zu analysieren und dysfunktionales Verhalten zu bemerken. Grundsätzlich kann dies für den Patienten eine Chance darstellen, seine Selbstbeobachtung und Introspektion in einem funktionalen Sinne zu verfeinern und 
zu einem besseren Verständnis der eigenen Person zu gelangen mit dem Ziel, unerwünschte bzw. belastende Zustände langfristig selber beeinflussend verändern zu können. Das Beispiel einer Verhaltensanalyse ist in Tabelle 4 dargestellt.

Die Kontingenz der Stimulus-Reaktions-Verknüpfung in diesem Fallbeispiel kann als sehr hoch eingestuft werden, da der Patient nach jeder von ihm als potenziell gefährlich eingestuften Situation eine starke Vermeidungsreaktion (beispielsweise sich nach Kontakt mit «infektiösem» Material nicht mehr zu berühren) bzw. ein Sicherheitsverhalten (ritualisiertes Händewaschen) zeigt, das kurzfristig zu einer Erleichterung führt. Langfristig belasten ihn jedoch negative Konsequenzen. Üblicherweise werden die kurzfristigen «Vorteile» des dysfunktionalen Verhaltens als derart sicherheitsspendend bzw. beruhigend erlebt, dass das Problemverhalten nur widerwillig verändert wird; denn die Reaktion (kogn, emot, phys, mot) auf einen subjektiv bedrohlichen Stimulus wird meist als sehr aversiv erlebt, sodass die Motivation, diesen Zustand schnellstmöglich zu beenden, sehr hoch ist. Dementsprechend wird dysfunktionales Verhalten häufig trotz kognitiver Einsicht in die Dysfunktionalität sowie der langfristig erlebten negativen Konsequenzen durch das kurzfristige Erleben von Erleichterung aufrecht erhalten (vgl. Abschnitt zum S-O-R-K-C-Modell).

Ist das Analysieren des eigenen Verhaltens erst einmal eingeübt, fällt es dem Patienten einfacher, die eigenen StimulusReaktions-Muster zu durchschauen und die eigenen Reaktionen auf den unterschiedlichen Ebenen nachzuvollziehen. Erst das Verständnis solcher Abfolgen kann zu einem bewussteren Umgang mit subjektiv bedrohlichen Situationen und letztlich zur Unterbrechung bzw. Veränderung dysfunktionalen Verhaltens führen.

Eine durch Verhaltensanalysen eingeübte Selbstbeobachtung kann zudem für die detaillierte Definition der konkreten Therapieziele genutzt werden, indem diese explizit und überprüfbar ausformuliert werden.

\section{Der Mehrwert einer Synthese störungsorientierten}

Wissens und individueller Verhaltensanalyse als Grundlage der Therapieindikation

Wie bereits Caspar und Grawe [1996] erörtert haben, schließen sich eine individuelle Verhaltensanalyse und allgemeines, störungsorientiertes Wissen nicht aus, sondern ergänzen sich wertvoll. So kann einerseits das störungsorientierte Wissen die Einordnung von Symptomen und Verhaltensweisen stark erleichtern und die Effizienz im therapeutischen Prozess steigern. Andererseits kann mit Hilfe der Verhaltensanalyse der Gefahr entgegengetreten werden, dass Patienten, die nicht in das störungsorientierte Schema passen, zu allgemein behandelt werden und sich dadurch nicht wahrgenommen und unverstanden fühlen. Eine Verhaltensanalyse, die subjektive und individuelle Funktionsmuster zu Tage bringt, kann hier das empirisch überprüfte Wissen ergänzen und es dem Patienten psychoedukativ zugänglich machen. Exempla- risch soll anhand des geschilderten Fallbeispiels ein kurzer störungsorientierter Hintergrund zu Zwangsstörungen und Phobien gegeben werden, um in einem nächsten Schritt die Relevanz der Synthese von empirisch überprüftem Wissen und individuellen Angaben im Rahmen der Therapieplanung zu erörtern.

Zwangsstörungen kommen gemäß Emmelkamp und van Oppen [2000] in der Bevölkerung mit einer durchschnittlichen Lebenszeitprävalenz von 2,3\% vor. Dabei sind etwa ebenso viel Männer wie Frauen betroffen, wobei Männer eher Kontrollzwänge entwickeln, während Frauen eher von Waschzwängen betroffen sind. Eine Zwangsstörung entsteht üblicherweise zwischen dem 20. und 25. Lebensjahr und kann gemäß des Zwei-Faktoren-Modells nach Mowrer [1939] durch ein spezifisches Ereignis ausgelöst werden. Dabei wird die Entstehung einer Zwangsstörung als Phänomen einer klassischen Konditionierung gesehen, während die Aufrechterhaltung auf operanten Konditionierungsmechanismen beruht. Letztlich kann man jedoch - wie bei den meisten psychischen Erkrankungen - ein biopsychosoziales Entstehungsmodell heranziehen. Komorbiditäten sind im Bereich von Zwangsstörungen äußerst häufig: Etwa 50\% aller Zwangspatienten weisen eine komorbide spezifische Phobie auf (bei Waschzwang häufig in der Kombination mit Erkrankungsphobie). Ebenso treten vermehrt affektive Störungen - insbesondere depressive Episoden - auf. Die Wahrscheinlichkeit, dass eine Depression reaktiv auf eine Zwangserkrankung folgt, scheint 3-mal größer zu sein als im umgekehrten Fall [Emmelkamp und van Oppen, 2000; Reinecker, 2009]. Auch sind Persönlichkeitsstörungen, Essstörungen und Suchterkrankungen häufig komorbid vorhanden. Ohne adäquate Behandlung besteht bei einer Zwangsstörung die Gefahr einer Chronifizierung. Des Weiteren findet das Vermeidungsverhalten bei einer Zwangsstörung häufig in einer (motorisch) aktiven Form statt, also beispielsweise Händewaschen, putzen, etc.; im Gegensatz dazu ist das Vermeidungsverhalten bei einer spezifischen Phobie eher passiv (d.h. Situationen/Dinge/Gedanken werden spezifisch vermieden) [Reinecker, 2009]. Auf biologischer Ebene wird vermutet, dass Angststörungen mit der Dysregulation diverser Neurotransmittersysteme in Zusammenhang stehen, insbesondere dem GABAergen, dem noradrenergen sowie dem serotonergen System (vermutet wird eine Überempfindlichkeit der 5-HT-Rezeptoren). Aus diesem Grund können Antidepressiva, die spezifisch auf das serotonerge System Einfluss nehmen, in der Behandlung von Zwangsstörungen effektiv sein [Emmelkamp und van Oppen, 2000].

Auf den diskutierten Fall bezogen, weicht der Patient zwar hinsichtlich des Erstauftretens der Symptomatik in Geschlecht und Alter vom Durchschnitt ab, zeigt aber eine typische komorbide Erscheinung von Waschzwang und damit eine zusammenhängende spezifische Erkrankungsphobie. Auch scheinen bei ihm die erkennbare reaktive, depressive Symptomatik sowie die Chronifizierung seines Zustands häufige Phänomene dieses Störungskomplexes zu sein. Ebenso ist 
die Ätiologie in diesem Fall aufgrund einer klassischen Konditionierung in einer spezifischen Situation (Wurmerkrankung nach Auslandaufenthalt) sowie der Aufrechterhaltung der Störung durch instrumentelle Konditionierung (beispielsweise die empfundene Erleichterung nach dem Händewaschen, nachdem ein subjektiv infizierter Gegenstand berührt wurde) gut erklärbar. Das störungsorientierte Wissen im Bereich der Ätiologie und der aufrechterhaltenden Mechanismen kann demzufolge der Einzelfallanalyse als Heuristik dienen.

\section{Therapieplanung aufgrund des störungsorientierten}

Wissens und der individuellen Verhaltensanalyse

Bei Zwangsstörungen ist eine kognitive Verhaltenstherapie die Methode der Wahl; je nach Art und Ausprägung der Zwänge auch in Kombination mit einer psychopharmakologischen Behandlung. Dabei ist das Eruieren automatischer Gedanken sowie die Modifikation zentraler Grundannahmen wesentlich [Emmelkamp und van Oppen, 2000]. Gemäß Hautzinger [2010] sind unter den psychologischen Behandlungsformen von Depressionen bei mittelschweren unipolaren Depressionen strukturierte, fokussierte Psychotherapien indiziert; gegebenenfalls in Kombination mit einer Psychopharmakotherapie. Auf die depressive Symptomatik des Patienten wird an dieser Stelle nicht näher eingegangen. Es wäre jedoch eine Verbesserung bei Abklingen der beiden erstgenannten Störungen zu vermuten.

Bereits anhand der ersten Erhebung von anamnestischen Daten sowie einer ausführlichen Diagnostik und Exploration können Verhaltensanalysen durchgeführt und Störungsmodelle sowie Zusammenhänge zwischen der spezifischen Phobie und dem Waschzwang erörtert und individualisiert adaptiert werden. In einem ersten Schritt des darauffolgenden Therapieprozesses definiert der Patient mit Unterstützung des Therapeuten seine Therapieziele und erstellt nach Anleitung Angsthierarchien bezüglich seiner spezifischen Erkrankungsangst (spezifische Phobie) auf einer Skala von 1 (= keine Angst) bis 10 (= maximale Angst). Ebenso soll der Drang, sich in der Folge als Vermeidungsreaktion zu waschen, eingestuft werden. Gleichzeitig können weiterhin Selbstbeobachtungen sowie das Führen eines Tagebuchs auch während der weiteren Therapiephasen hilfreich sein, um die Veränderung über die Zeit hinweg sowie die Erreichung der angestrebten Ziele zu überprüfen.

Aufgrund des störungsorientierten Wissens kann davon ausgegangen werden, dass in diesem Fall die spezifische Phobie und der Waschzwang nicht voneinander unabhängig sind, sondern chronologisch aufeinander im Rahmen einer raumund zeitkontingenten Stimulus-Reaktion-Verkettung mit anschließendem Vermeidungs- bzw. Sicherheitsverhalten auftreten. Aufgrund dieses Wissens können konkrete In-vivo-Expositionen geplant werden, die einerseits die beiden Störungskomplexe einzeln, aber eben auch vor allem in ihrer Verknüpfung bearbeiten. Durch die Informationen aus den erarbeiteten Verhaltensanalysen können die Situationen sehr individuell ausgewählt werden (beispielsweise das Berühren von Erdbeeren in der Obstabteilung mit anschließender Reaktionsverhinderung, d.h. dem Nicht-Waschen der Hände). Dabei soll gleichzeitig, wie bei Expositionen üblich, die Veränderung der Angstintensität über die Zeit hinweg beschrieben werden, bzw. es wird erlebt, wie die Habituation funktioniert.

Da die Ätiologie im Fall des Patienten auch eine bereits früh in der Kindheit gelernte Komponente aufweist (übertriebene Hygiene im Elternhaus) und die perfektionistische Persönlichkeitsstruktur des Patienten die Symptomatik zu aggravieren scheint, sollten diese Aspekte in der Therapie im Sinne einer erhöhten Nachhaltigkeit der therapeutischen Zielerreichung ebenso bearbeitet werden. Auch hier können spezifische Verhaltensanalysen erarbeitet werden, um dem Patienten Zusammenhänge über das eigene innerpsychische Funktionieren zugänglich zu machen. Zur Unterstützung könnten hier auch Worst-Case-Szenarien als Stimulus sowie Risikoabschätzungen für das tatsächliche Eintreffen der Befürchtung besprochen werden.

\section{Ausblick und Fazit}

Trotz der Kritik an der Verhaltensanalyse als Methode lässt sich festhalten, dass sie vielseitig und für den therapeutischen Prozess fruchtbar eingesetzt werden kann. Obwohl individuelle Verhaltensanalysen bei Störungen mit bereits empirisch validierten Ätiologie- bzw. Erklärungsmodellen nicht als entscheidende Faktoren für den Therapieerfolg angesehen werden [z.B. Eifert, 2005], bemerken Tuschen-Caffier und von Gemmeren [2009] treffend, dass im Rahmen einer störungsfokussierten Diagnostik idealtypisch von einer Einzelerkrankung ausgegangen und in der Folge nicht dem Umstand Rechnung getragen wird, dass Patienten im klinischen Alltag oftmals komplexe, komorbide Störungen aufweisen, die sich nicht immer mit spezifischen und empirisch überprüften, störungsorientierten Behandlungsmethoden therapieren lassen. Des Weiteren bemerken die Autorinnen, dass grundsätzlich neben den störungsfokussierten Faktoren auch interpersonellen Aspekten Beachtung geschenkt werden sollte. Diesbezüglich könnte die 3. Welle der Verhaltenstherapie, die über die behavioralen und kognitiven Aspekte hinaus wieder vermehrt die emotional-regulatorischen Aspekte in den Mittelpunkt stellt, in den Vordergrund rücken. Auf der Verhaltenstherapie basierende, jedoch dynamischere, störungsübergreifende Therapieformen, wie beispielsweise die Schematherapie nach Young et al. [2003] oder die emotionsfokussierte Therapie nach Greenberg [2002], bringen zudem weitere, ergänzende Methoden hervor, welche die interpersonellen Aspekte stärker berücksichtigen.

Zusammenfassend kann konstatiert werden, dass die Verhaltensanalyse zur Ausarbeitung eines hypothetischen Bedin- 
gungsmodells herangezogen werden und gemeinsam mit störungsorientiertem und empirisch überprüftem Wissen zur effizienten Therapieplanung dienen kann. Kontrollierte Studien sind notwendig, um empirische Aussagen über die Effektivität von individualisierten Varianzanalysen im therapeutischen Prozess treffen zu können. Als Instrument zur Erklärung des individuellen Problemverhaltens bzw. der funktionalen $\mathrm{Zu}$ sammenhänge des Problemverhaltens mit den vorausgehenden und nachfolgenden Bedingungen ist eine Verhaltensanalyse jedoch zweifelsohne hilfreich. Für den Patienten bietet die Verhaltensanalyse zudem unter Umständen eine einfache
Möglichkeit, strukturierte Einsicht in komplexe Prozesse der eigenen Funktionsweise zu erhalten. Um empirische Aussagen über die Effektivität von individualisierten Varianzanalysen im therapeutischen Prozess treffen zu können, wären jedoch kontrollierte Studien erforderlich.

\section{Disclosure Statement}

Die Autoren erklären hiermit, dass kein Interessenskonflikt besteht.

\section{Literatur}

Bartling G, Echelmeyer L, Engberding M: Problemanalyse im psychotherapeutischen Prozess. Leitfaden für die Praxis. Stuttgart, Kohlhammer, 1980/2008.

Caspar F: Was ist aus der guten alten Verhaltensanalyse geworden?; in ders. (ed): Psychotherapeutische Problemanalyse. Tübingen, DGVT, 1996, pp 7-43.

Caspar F, Goldfried MR: Beziehungen und Probleme verstehen: Eine Einführung in die psychotherapeutische Plananalyse. Bern, Huber, 2007.

Caspar F, Grawe K: Was spricht für, was gegen individuelle Fallkonzeption?; in Caspar F (ed) Psychotherapeutische Problemanalyse. Tübingen, DGVT, 1996, pp 65-85.

Emmelkamp MG, van Oppen P: Zwangsstörungen. Göttingen, Hogrefe, 2000.

Eifert GH: Individuelle versus standardisierte Therapie. Erkenntnisse und neue Fragestellungen; in Kosfelder J, Michalak J, Vock S, Willutzki U (eds): Fortschritte der Psychotherapiforschung. Göttingen, Hogrefe, 2005, pp 197-216.

Evans RI, Rozelle RM, Mittelmark MB, Hansen WB, Bane AL, Havis J: Deterring the onset of smoking in children: knowledge of immediate physiological effects and coping with peer pressure, media pressure, and parent modeling. J Appl Soc Psychol 1978;8:126-135.

Grawe K: Implikationen und Anwendungsmöglichkeiten der vertikalen Verhaltensanalyse für die Sichtweise und Behandlung psychischer Störungen (Forschungsbericht Nr. 5). Universität Bern, 1982.
Grawe K, Caspar F (eds): Die Plananalyse als Konzept und Instrument für die Psychotherapieforschung. Göttingen, Hogrefe, 1984.

Greenberg L: Emotion-Focused Therapy: Coaching Clients to Work Through Feelings. Washington, American Psychological Association Press, 2002.

Hautzinger M: Akute Depression. Göttingen, Hogrefe, 2010.

Hautzinger M: Mirko-Verhaltensanalyse; in Linden M, Hautzinger M (eds): Verhaltenstherapiemanual. Berlin, Springer, 2011, pp 217-221.

Kanfer FH, Reinecker H, Schmelzer D: Selbstmanagement-Therapie: Ein Lehrbuch für die klinische Praxis. Heidelberg, Springer, 2006.

Kanfer FH, Saslow G: Behavioral diagnosis; in Franks CM (ed): Behavior Therapy: Appraisal and Status. New York, McGraw-Hill, 1969.

Knafla I, Ehlert U: Verhaltensanalyse. Psychotherapeut 2001;46:145-153.

Lindsley OR: Direct measurement and prosthesis of retarded behavior. J Educ 1964;147:62-81.

Lundberg U, Wright JM, Frankenhabuser M, Olson UJ: Involvement in four future events as a function of temporal distance. Scand J Psychol 1975;16:2-6.

Meinlschmidt G, Tegethoff M: Psychotherapeutische Fallberichte bei Erwachsenen; in Magraf J, Schneider S (eds): Lehrbuch der Verhaltenstherapie. Band 1. Grundlagen, Diagnostik, Verfahren, Rahmenbedingungen. Heidelberg, Springer, 2009, pp 900-923.
Mowrer OH: A stimulus-response analysis of anxiety and its role as a reinforcing agent. Psychol Rev 1939;46:553-565.

Narciss S: Verhaltensanalyse und Verhaltensmodifikation auf der Basis lernpsychologischer Erkenntnisse; in Wittchen HU, Hoyer J (eds): Klinische Psychologie und Psychotherapie. Berlin, Springer, 2011, pp 419-433.

Neudeck P, Mühlig S: Therapie-Tools Verhaltenstherapie: Therapieplanung, Probatorik, Verhaltensanalyse. Weinheim, Beltz, 2013.

Reinecker H: Zwangshandlungen und Zwangsgedanken. Göttingen, Hogrefe, 2009.

Schulte D: Therapieplanung. Göttingen, Hogrefe, 1996.

Schulte D: Verhaltenstherapeutische Diagnostik; in Reinecker H (ed): Lehrbuch der Verhaltenstherapie. Tübingen, DGVT, 1999.

Tuschen-Caffier B, von Gemmeren B: Problem- und Verhaltensanalyse; in Magraf J, Schneider S (eds): Lehrbuch der Verhaltenstherapie. Band 1. Grundlagen, Diagnostik, Verfahren, Rahmenbedingungen. Heidelberg, Springer, 2009, pp 364-375.

Young J, Klosko JS, Weishaar ME: Schema Therapy. A Practitioners Guide. New York, Guilford Press, 2003.

Zaby A, Heider J: Verhaltenstherapeutische Diagnostik; in Senf W, Broda M (eds): Praxis der Psychotherapie. Ein integratives Lehrbuch. Stuttgart, Thieme, 2012, pp 293-303. 